\title{
Case Report: Malignant Ventricular Arrhythmias Mimicking Acute Coronary Syndrome in Chagas Disease
}

\author{
Renata Junqueira Moll-Bernardes, ${ }^{1 \star}$ Roberto Magalhães Saraiva, ${ }^{2}$ Renée Sarmento de Oliveira, ${ }^{1}$ Martha Valéria Tavares Pinheiro, ${ }^{1}$ \\ Gabriel Cordeiro Camargo, ${ }^{1,3}$ Adriana Soares Xavier de Brito, ${ }^{1,3}$ Sergio Altino de Almeida, ${ }^{1}$ Fabio Paiva Rossini de Siqueira, ${ }^{1}$ \\ Fernanda de Souza Nogueira Sardinha Mendes, ${ }^{2}$ Rodrigo Minati Barbosa, ${ }^{3}$ Sergio Salles Xavier, ${ }^{2}$ \\ Paulo Henrique Rosado de Castro, ${ }^{1}$ and Andréa Silvestre de Sousa ${ }^{1,2}$ \\ ${ }^{1}$ D'Or Institute for Research and Education (IDOR), Rio de Janeiro, Brazil; ${ }^{2}$ Evandro Chagas National Institute of Infectious Diseases, Oswaldo Cruz \\ Foundation, Rio de Janeiro, Brazil; ${ }^{3}$ National Institute of Cardiology (INC), Rio de Janeiro, Brazil
}

\begin{abstract}
Chronic Chagas heart disease has different clinical manifestations including arrhythmias, heart failure, and stroke. Chest pain is one of the most common symptoms and when associated with changes in the electrocardiogram, such as T-wave changes, electrically inactive areas, and segmental wall motion abnormalities, may lead to a misdiagnosis of acute coronary syndrome (ACS). Here, we describe two patients with Chagas heart disease and syncope due to sustained ventricular tachycardia who were misdiagnosed with ACS, and discuss the role of novel imaging modalities in the differential diagnosis and risk stratification.
\end{abstract}

\section{CASE REPORT SECTION}

Case 1. A 54-year-old woman was admitted with a history of syncope, chest pain, and palpitations. She was born in the state of Minas Gerais, Brazil, and has been followed up for 1 year in Rio de Janeiro, and was diagnosed with Chagas heart disease complicated by heart failure. In the emergency department, her blood analysis evidenced a high troponin serum level and electrocardiogram (ECG) showed sinus rhythm, left anterior fascicular block, and primary T-wave changes. Associated acute coronary syndrome (ACS) was suspected, and she underwent angiography that revealed normal coronaries. Two days later, she presented sustained ventricular tachycardia (VT), reversed with amiodarone (Figure 1A). Echocardiogram depicted severe left ventricular (LV) systolic dysfunction with apical aneurysm and extensive changes in segmental contractility (Figure 1B). Cardiac magnetic resonance (CMR) with late gadolinium enhancement (LGE) revealed severe LV systolic dysfunction; apical transmural fibrosis; and anterior, lateral, and inferior mesoepicardial fibrosis (Figure 1C); T1 mapping showed diffuse interstitial fibrosis, with elevated native T1 and extracellular volume (Figure 1D). Positron emission tomography/ computed tomography (PET/CT) showed an increased uptake of fluorine-18-labeled 2-deoxy-2-fluoro-d-glucose ( ${ }^{18} \mathrm{~F}-\mathrm{FDG}$ ) in mid-basal anterior, lateral, and septal walls and a decreased uptake in the apical region (aneurysm). An implantable cardioverter defibrillator (ICD) was successfully implanted; she was released and now is currently asymptomatic.

Case 2. A 68-year-old man presented with an episode of syncope and VT, which was reversed with electrical cardioversion, during a recent trip to his hometown in Ceará, Brazil. He has been followed up because of Chagas heart disease in Rio de Janeiro and had no previous cardiac symptoms. Electrocardiogram showed sinus rhythm, low voltage, complete right bundle block, left anterior fascicular block, anterolateral electrically inactive area, and primary T-wave changes (Figure 2A). Echocardiogram (Figure 2B)

\footnotetext{
*Address correspondence to Renata Junqueira Moll-Bernardes, D'Or Institute for Research and Education, Rua Diniz Cordeiro, 30, 3rd floor, Botafogo, Rio de Janeiro, Brazil 22281-100. E-mail: renata.moll@ idor.org
}

showed moderate LV systolic dysfunction with apical aneurysm. After cardioversion, he underwent coronary angiography, and although only a myocardial bridge with coronary spasm was present in the anterior descending artery, an angioplasty with stent implantation was performed. Eight days later, he had a new episode of VT (Figure 2A) with spontaneous reversal and an ICD was indicated. Cardiac magnetic resonance demonstrated apical aneurysm and inferolateral mesoepicardial fibrosis (Figure 2C). T1 mapping showed diffuse interstitial fibrosis with elevated native $\mathrm{T} 1$ and extracellular volume (Figure 2D). Technetium-99m sestamibi single-photon emission computed tomography evidenced apical and inferolateral hypoperfusion in rest. After ICD implantation, he was released with no symptoms using regular medications plus amiodarone and clopidogrel.

\section{DISCUSSION}

Patients with chronic Chagas heart disease often present with angina-like symptoms associated with ischemic changes in ECG and segmental contractile dysfunction, which makes the differential diagnosis of coronary heart disease quite difficult. ${ }^{1}$ Although the possibility of associated lesions should be considered, these patients usually have normal coronaries, and their ischemia-like symptoms can be explained by coronary microvascular abnormalities. The appropriate diagnosis should be pursued to avoid overtreatment, as in case 2 who underwent a coronary stent implantation.

In the emergency department, patients with Chagas heart disease may present with syncope due to ventricular arrhythmia or even sudden cardiac arrest (SCA), mimicking an ACS. Scar-mediated reentry is the most common mechanism for VT in Chagas heart disease and occurs as a result of myocardial fibrosis, which is a strong predictor of major adverse outcomes. ${ }^{2,3}$ The aforementioned cases illustrate the use of CMR with LGE to help in the differential diagnosis. In Chagas heart disease, the pattern of LGE is usually mesoepicardial or transmural and the most frequent localizations are apical or inferobasal segments of the LV, which differs from ischemic cardiomyopathies, in which LGE matches a coronary territory distribution with a subendocardial fibrosis pattern. 

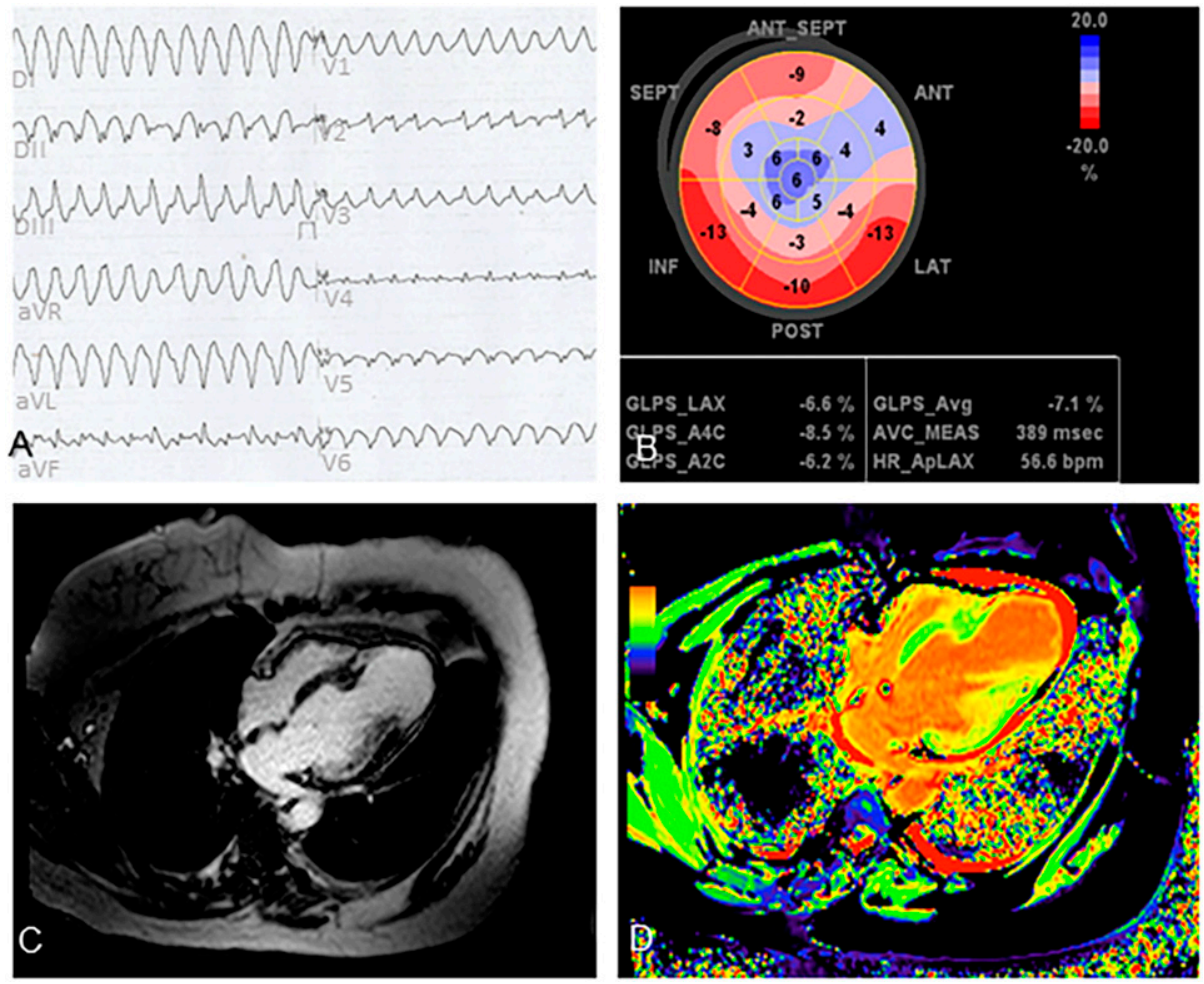

FIGURE 1. Multimodality imaging assessment (case 1). (A) Electrocardiographic recording of sustained ventricular tachycardia. (B) Echocardiography with strain analysis evidences apical dyskinesis and anterior akinesis. (C) Cardiac magnetic resonance (CMR) image depicts mesoepicardial late gadolinium enhancement (LGE) in the left ventricular (LV) lateral wall and transmural LGE involving the LV apex with aneurysm formation. (D) CMR T1 mapping shows longer native T1 and elevated extracellular volume. This figure appears in color at www.ajtmh.org.
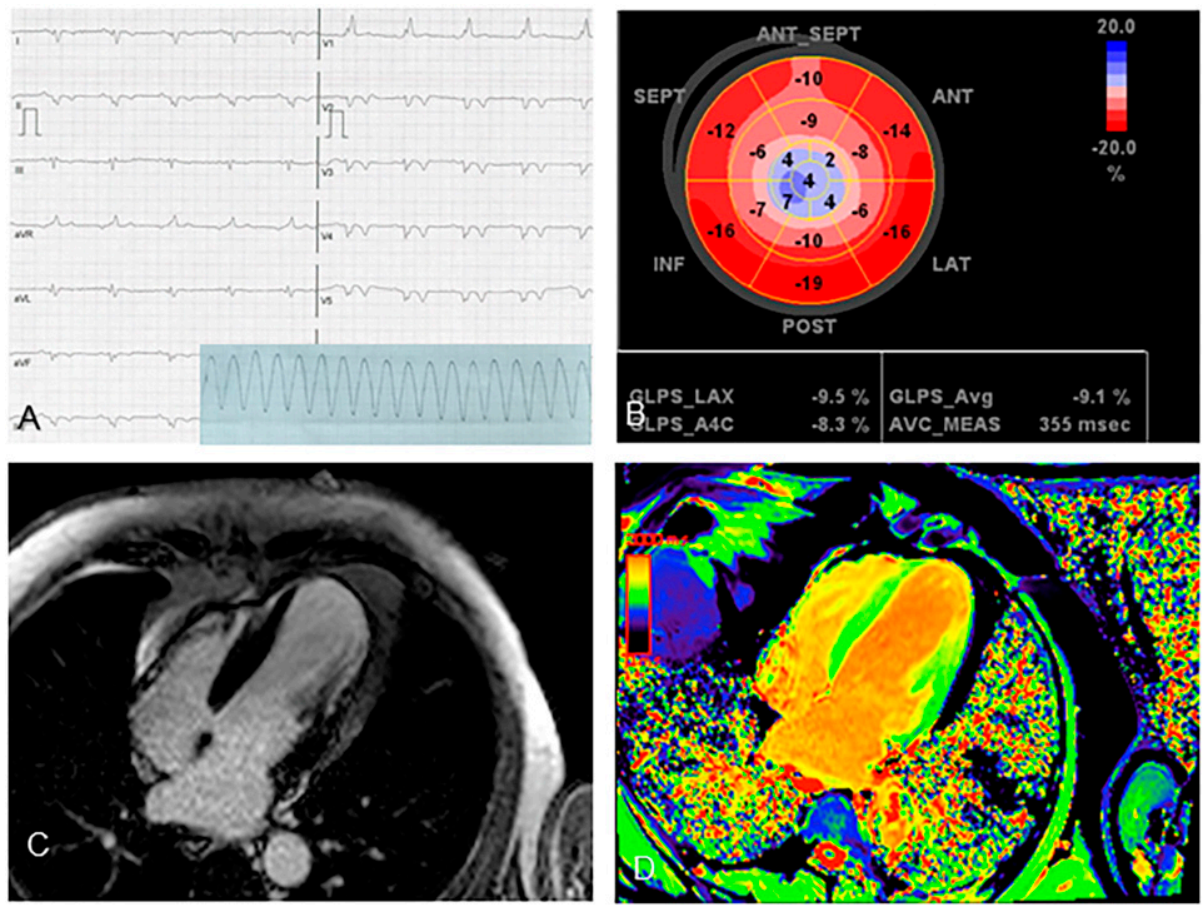

FIGURE 2. Multimodality imaging assessment (case 2). (A) Electrocardiogram shows sinus rhythm, low voltage, complete right bundle block, left anterior fascicular block, anterolateral electrically inactive area, and primary T-wave changes. Details in the bottom-right corner depict the episode of sustained ventricular tachycardia. (B) Echocardiography with strain analysis shows apical dyskinesis. (C) Cardiac magnetic resonance (CMR) demonstrated apical aneurysm and inferolateral mesoepicardial fibrosis with late gadolinium enhancement. (D) CMR T1 mapping shows longer native T1 and elevated extracellular volume. This figure appears in color at www.ajtmh.org. 
Persistent subclinical inflammation in areas adjacent to fibrotic regions may act as a trigger for ventricular arrhythmia, which was evidenced in case 1 . Our finding is in accordance with previous reports which suggest that inflammation could be detected with ${ }^{18} \mathrm{~F}-\mathrm{FDG}$ PET/CT in Chagas patients. ${ }^{4,5}$ Better understanding of the role of inflammation detected by PET/CT may provide new parameters for risk stratification for SCA.

Chagas heart disease must be considered in all patients from endemic areas with syncope and VT, and ICD implantation should be indicated as a secondary preventive strategy of SCA in these cases. Ideally, the risk of sudden cardiac death should be evaluated in earlier stages of the disease, using new imaging methods such as CMR and PET/CT, for the implementation of primary preventive strategies. Unfortunately, despite its high mortality, there is no clear recommendation for early ICD implantation in patients with Chagas heart disease in current guidelines.

Recent advances in cardiac imaging may help us in the differential diagnosis between Chagas heart disease and ACS, and also in risk stratification for SCA. Both reported cases illustrate that even with previous diagnosis of Chagas heart disease, patients with syncope and VT are frequently considered as having ACS, revealing the lack of awareness of health professionals regarding this neglected disease.

Received December 10, 2019. Accepted for publication December 26, 2019.

Published online February 10, 2020.

Financial support: This work was supported by the D'Or Institute for Research and Education (IDOR), Rio de Janeiro, and the Oswaldo Cruz Foundation, INOVA (VPPCB-007-FIO-18-2-10-30), Rio de Janeiro, Brazil.
Authors' addresses: Renata Junqueira Moll-Bernardes, Sergio Altino de Almeida, Fabio Paiva Rossini de Siqueira, and Paulo Henrique Rosado de Castro, D'Or Institute for Research and Education (IDOR), Rio de Janeiro, Brazil, E-mails: renata.moll@idor.org, reneemedicina@ yahoo.com.br, martha.pinheiro2@gmail.com, altino.sergio@gmail. com, fabioprs@gmail.com, and phrosado@gmail.com. Roberto Magalhães Saraiva, Fernanda de Souza Nogueira Sardinha Mendes, and Sergio Salles Xavier, Evandro Chagas National Institute of Infectious Diseases, Oswaldo Cruz Foundation, Rio de Janeiro, Brazil, E-mails: roberto.saraiva@ini.fiocruz.br, nandasardinha@gmail.com, and sergiosallesx@gmail.com. Gabriel Cordeiro Camargo and Adriana Soares Xavier de Brito, D'Or Institute for Research and Education (IDOR), Rio de Janeiro, Brazil, and National Institute of Cardiology (INC), Rio de Janeiro, Brazil, E-mails: gabccamargo@gmail.com and adrijsoares@hotmail.com. Rodrigo Minati Barbosa, National Institute of Infectious Diseases, Oswaldo Cruz Foundation, Rio de Janeiro, Brazil, E-mail: rodrigobarbosa@bighost.com.br. Andréa Silvestre de Sousa, D'Or Institute for Research and Education (IDOR), Rio de Janeiro, Brazil, and Evandro Chagas National Institute of Infectious Diseases, Oswaldo Cruz Foundation, Rio de Janeiro, Brazil, E-mail: andrea.silvestre@globo.com.

\section{REFERENCES}

1. Nunes MCP et al., 2018. Chagas cardiomyopathy: an update of current clinical knowledge and management: a scientific statement from the American Heart Association. Circulation 138: e169-e209.

2. Volpe GJ et al., 2018. Left ventricular scar and prognosis in chronic Chagas cardiomyopathy. J Am Coll Cardiol 72: 2567-2576.

3. Senra T, lanni BM, Costa AC, Mady C, Martinelli-Filho M, Kalil-Filho $R$, Rochitte $C E, 2018$. Long-term prognostic value of myocardial fibrosis in patients with Chagas cardiomyopathy. $\mathrm{J} \mathrm{Am}$ Coll Cardiol 72: 2577-2587.

4. Garg G, Cohen S, Neches R, Travin MI, 2016. Cardiac 18 F-FDG uptake in Chagas disease. J Nucl Cardiol 23: 321-325.

5. Shapiro H, Meymandi S, Shivkumar K, Bradfield JS, 2017. Cardiac inflammation and ventricular tachycardia in Chagas disease. Heartrhythm Case Rep 3: 392-395. 